\title{
Determination of Bergenin in Sechangzhixiesan by High Performance Capillary Electrophoresis
}

\author{
Yun-Chen Zhang ${ }^{a}$, Ting-Ting Huang ${ }^{b}$, Yang $X^{c}{ }^{c}$, Hai-Xing Liu ${ }^{*}, d$ \\ Chemistry \& Chemical and Environmental Engineering College, Weifang University, Weifang \\ 261061, P.R. China \\ a4_haixingliu@tom.com, b2_haixingliu@tom.com, c3_haixingliu@tom.com, dhaixingliu@tom.com,
}

Keywords: capillary electrophoresis, bergenin, Sechangzhixiesan

\begin{abstract}
This paper presents the determination of bergenin content of Sechangzhixiesan by high performance capillary electrophoresis (HPCE) method. The borax solution as buffer solution was chosen, and its concentration was $20 \mathrm{mmol} / \mathrm{L}$ at a constant voltage of $20 \mathrm{kV}$ and injecting time of 10s. Measured bergenin content in Sechangzhixiesan was $8.21 \mathrm{mg} / \mathrm{g}(\mathrm{RSD}=3.8 \%)(\mathrm{n}=8)$. The recovery was in the range of $86.3 \%-92.3 \%(n=4)$. This method is specific, simple and rapid and accurate, which is suitable for the detection of the content of bergenin in Sechangzhixiesan.
\end{abstract}

\section{Introduction}

Sechangzhixiesan is consists of bentonite and rock Vedas. It has the function of convergence anti-diarrhea and strengthening the spleen and regulating the stomach. It is used for the treatment of spleen stomach qi deficiency, diarrhea, acute and chronic enteritis, enteritis anaphylactica, indigestion, intestinal disorders, etc [1]. Bergenin is the active ingredient of saxifragaceae plants, such as purple bergenia herb, featherleaf rodgersflower, astilbe, et al. It is white loose needle crystal or crystalline powder, micro gas, bitter, In case of light and heat discoloration. It is soluble in methanol and slightly soluble in water, acetone or ethanol. The research showed bergenin has the effect of relieve cough, immune enhancement, promoting the recovery of diseased tissue, antiviral HIV, anticoagulant and liver protection [2]. Li et al [3] developed an HPLC method for determination of bergenin in Zhixie capsules. The ODS-C18 column was used. The mobile phase composed of methanol-water (20:80). The detection wavelength was $275 \mathrm{~nm}$. Chen et al [4] developed aquantitative method for determination of bergenin, 11-o-galloyl bergenin and 11-o-(4-hydroxyl benzoyl) bergenin in Cissus pteroclada Hayata with HPLC, the expermient is studied in gradient separations with a column SinoChrom ODS-BP C18 $(5 \mu \mathrm{m}, 4.6 \mathrm{~mm} \times 200 \mathrm{~mm})$ and solvent consisted of MeCN-0.1\% formic acid. The flow rate is $0.8 \mathrm{~mL} / \mathrm{min}$. The wavelength was set at $275 \mathrm{~nm}$. The simple method based on matrix solid-phase dispersion with molecularly imprinted polymers as the sorbent was explored by Zhu et al [5] for selective extraction of bergenin from Ardisia japonica. The MIPs were synthesized using bergenin as a template molecule, methacrylic acid as a functional monomer, and ethylene glycol dimethacrylate as a crosslinking agent. The polymers have been characterized by scanning electron microscopy and Fouriertransform infrared spectrometry. The maximum extraction yield of bergenin was studied. The optimized extraction conditions as: $2 / 1$ as the ratio of MIPs to the sample; $8 \mathrm{~min}$ as the dispersion time; $10 \%$ aqueous methanol as a washing solvent and methanol-acetic acid ( $99: 1, \mathrm{~V} / \mathrm{V})$ as an elution solvent. The extract obtained was discussed by HPLC. The HPLC method for determining of bergeninum content in Ardision crispa (Thunb.) A.DC and KaiHou sword was established by Zhao et al [6]. The method was carried out on Luna-5u-c18 (2)-100A (150×4.6 mm, $5 \mu \mathrm{m})$ column with the mobile phase of methanol and water $(25: 75, \mathrm{v} / \mathrm{v})$. The detection wavelength was set at $274 \mathrm{~nm}$ and the flow rate was $1.0 \mathrm{~mL} / \mathrm{min}$. Optimal conditions of extracting bergenin from Astilbe chinensis using reflux method were established by Wang et al [7]. Single factor experiments and orthogonal text were performed to investigate the influences of the extraction factors on the yield of bergenin, which was determined by HPLC. The results indicated that the best optimal conditions were solvent $45 \%$ ethanol, time of refluxing 2.5 hours, ratio of material to 
solvent 1:20, and times of extraction 2. A chloroperoxidase( CPO) modified electrode was fabricated by Wang et al [8] using drop-coating the mixture solution of CPO and didodecyldimethylammonium bromide( DDAB) onto the glassy carbon electrode surface. Direct electron transfer of immobilized CPO was observed from cyclic voltammogram. The CPO modified electrode was further used as the working electrode for electrocatalytic chlorination of bergenin in $\mathrm{O}_{2}$-saturated bergenin solution containing potassium chloride. The chlorinated product was confirmed by HPLC-MS. In order to optimize the conditions of ultrasonic-assisted extraction method of bergenin from Rhizoma Bergeniae Scopulosae, on the base of single-factor experiment, an equation for the ultrasonic wave extraction was built by Wang et al [9]. The experiment indicated that ultrasonic extraction power, extraction time, ethanol concentration and extraction temperature had different effects on the bergenin extraction yield. The result showed that within tested level ranges, the importance of four process parameters affecting extraction yield of bergenin order were ultrasonic extraction temperature, ultrasonic power, ethanol concentration and ultrasonic extraction time. Five new bergenin analogs have been synthesized by Mao et al [10] via selective etherification and oxidation. Their anti-tumor activity were studied in vitro against Hela tumor cell lines. Five compounds were found to be active against hela. The interaction of Bergenin and DNA was studied by Liu et al [11] using UV spectrometry, viscosity method and fluorescence spectroscopy with Berberine(BR) as the fluorescent probe. The results indicated that at $\mathrm{pH} 7.4$ Tris- $\mathrm{HCl}$ buffer solution, the increase of the concentration of DNA caused red shift of the absorption peak and the decrease of the absorbance at $215 \mathrm{~nm}$ of Bergenin, which proved that there existed intercalative reaction between Bergenin and DNA; the fluorescence intensity of BR-DNA system was quenched when Bergenin was added and the quenching mechanism was judged as a static quenching. A simple and rapid method for analysis of bergenin in rabbit plasma with HPLC was established by Zheng et al [12], and then the pharmacokinetic of bergenin was discussed further in rabbits. Plasma samples which were extracted by ethyl acetate were used for analysis using HPLC. The mobile phase was methanol - water (22:78). After the bergenin was injected intravenously into rabbits, the blood concentration of drug can be detected, thus blood concentration time curves as well as pharmacokinetics parameters were worked out according to the PKS pharmacokinetics program. The optimize the extraction technology of bergenin in Rodgersia pinnata Hemsl was established by Wang et al [13]. The extraction technology of bergenin from Rodgersia pinnata Hemsl was optimized by orthogonal design taking the content of bergenin as index with the solvent concentration, the amount of solvent, the extraction time and extraction times as investigation factors. The optimal extraction technology of bergenin from Rodgersia pinnata Hemsl showed that the solvent was $60 \%$ methanol, solvent amount was $20 \mathrm{ml}$, extraction time was $20 \mathrm{~min}$ and extraction times was 3 . With the root of Ardisia crenata for the testing material, the optimization extraction conditions of bergenin were determined and analyzed systematically by $\mathrm{Hu}$ et al [14]. The results indicated that: (1) with the Sohlex method for extraction bergenin, the optimal conditions were as: the $95 \%$ alcohol concentration, $85^{\circ} \mathrm{C}$ of the extraction temperature, the rate of liquid to powder at $1: 12$, $6 \mathrm{~h}$ of the extraction time, then the extraction rate of bergenin was $2.30 \%$; (2) with the Ultrasonic for extraction method, the optimal conditions were as: the 95\% alcohol concentration, $50 \mathrm{~W}$ of the extraction power, 50 minutes of the extraction time, and the rate of liquid to powder at $1: 11$, then the extraction rate was achieved $2.54 \%$. In this paper, the bergenin content in Sechangzhixiesan was determined by High Performance Capillary Electrophoresis.

\section{Experimental section}

\subsection{Instruments and Reagents}

Experimental instruments: CL-1030-type high performance capillary electrophoresis (Beijing Cailu Scientific Instrument Co., Ltd.); HW2000-type chromatography workstation (Nanjing Qianpu Software Ltd.); Capillary ( $75 \mu \mathrm{m}$ inner diameter, $52 \mathrm{~cm}$ overall length, $44 \mathrm{~cm}$ effective length) from Hebei Yongnian Ruifeng Chromatographic Devices Co., Ltd.).

bergenin (Chinese Drugs and Biological Products); Sechangzhixiesan (Yunnan Shipurui 
Bioengineering Co., Ltd.); Other reagents used in the experiments were all analytical grade; Double-distilled water was used.

\subsection{Experimental Methods}

Before the start of the experiment, capillary was successively washed with $1 \mathrm{~mol} \cdot \mathrm{L}^{-1}$ hydrochloric acid solution, double-distilled water, $1 \mathrm{~mol} \cdot \mathrm{L}^{-1}$ sodium hydroxide solution, double-distilled water, buffer solution, each for $8 \mathrm{~min}$. After three times running, capillary was cleaned again using the above method.

Measurements were carded out at $20 \mathrm{kV}$ voltage and experimental temperature at $20^{\circ} \mathrm{C}$. UV detection wavelength was $275 \mathrm{~nm}$. Injection time was $10 \mathrm{~s}$ ( $7.5 \mathrm{~cm}$ height difference).

\subsection{Sample Preparation}

Sechangzhixiesan sample solution: Sechangzhixiesan powder was accurately weighed $3.1563 \mathrm{~g}$, added $20 \mathrm{~mL}$ water with $30 \%$ methanol, extracted time of $2 \mathrm{~h}$ at $60^{\circ} \mathrm{C}$, filtered, washed and set the volume to $50 \mathrm{~mL}$ that was the Sechangzhixiesan sample solution.

Bergenin standard solution: Bergenin was accurately weighed $5.4 \mathrm{mg}$, added $4 \mathrm{~mL}$ water with $30 \%$ methanol.

\section{Results and Discussion}

\subsection{Selection electrophoresis conditions}

Based on past experiment experience, we chose $20 \mathrm{mmol} / \mathrm{L}$ borax solution as a running buffer solution.

According to the literature, bergenin maximum absorption wavelength was at $275 \mathrm{~nm}$, so we chose the $275 \mathrm{~nm}$ detection wavelength.

\subsection{Quantitative analysis}

\subsubsection{Standard curve}

First, bergenin standard solution that the concentration were $1.350,0.675,0.338,0.169,0.085$ $\mathrm{mg} / \mathrm{mL}$ was prepared. Each standard solution was run for three times under the above electrophoresis conditions and the results averaged. The chromatogram of bergenin standard solution was showed in Figure 1. Taking concentration as the abscissa and peak area as the ordinate, the standard curve was drew. Linear regression equation of bergenin (peak area: $\mathrm{y} \mu \mathrm{V} \bullet \mathrm{s}$, density: $\mathrm{x}$ $\mathrm{mg} / \mathrm{mL})$ and the linear range was as follows: $\mathrm{Y}=-6404.58+129218.88 \mathrm{X}(\mathrm{r}=0.998), 0.085 \sim 1.350$ $\mathrm{mg} / \mathrm{mL}$. 


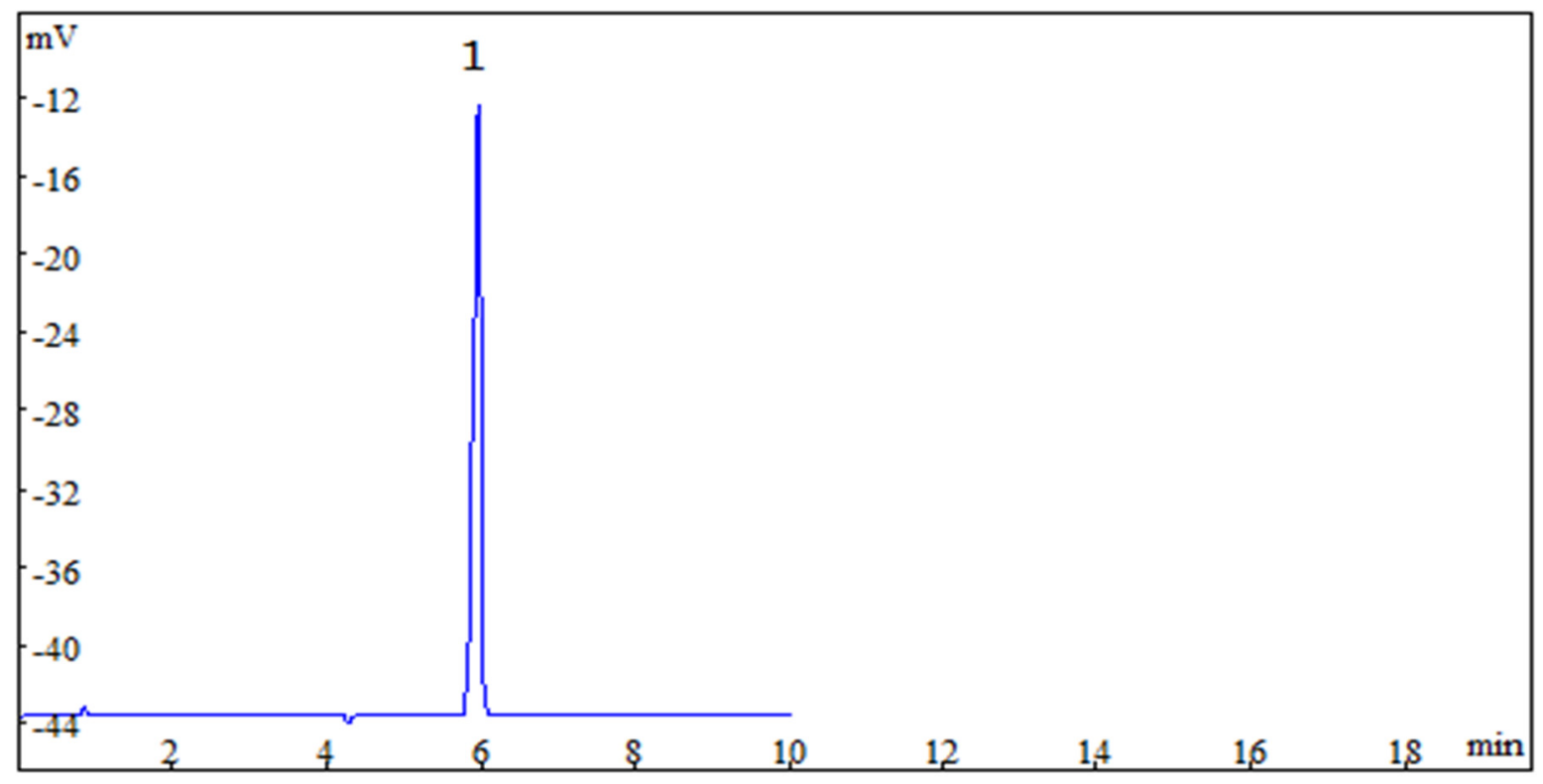

Fig.1 Electrophorogram of bergenin standard solution 1 - bergenin

\subsubsection{Precision test}

A bergenin standard solution precisely drew and continuously injected for sixt times under electrophoretic separation conditions, the RSD of bergenin peak area were $3.92 \%$, indicating good precision.

\subsubsection{Determination of sample content}

Under selected electrophoresis conditions, Sechangzhixiesan sample solution was run. Separation chromatogram of the Sechangzhixiesan sample solution was showed in Figure 2. Measured bergenin content in Sechangzhixiesan was $8.21 \mathrm{mg} / \mathrm{g}(\mathrm{RSD}=3.8 \%)(\mathrm{n}=8)$.

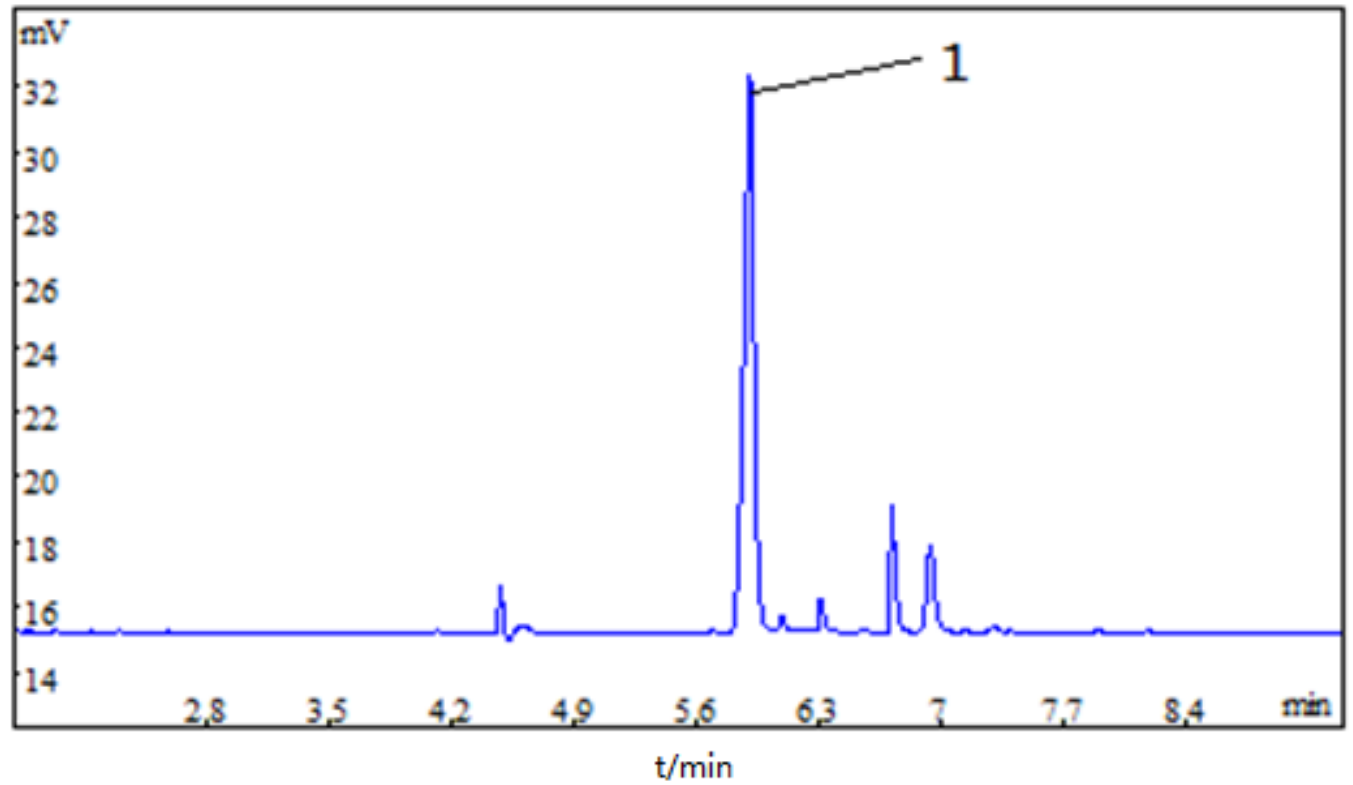

Fig.2 Electrophorogram of Sechangzhixiesan sample solution

1 - bergenin

\subsubsection{Recovery}

After determination for six times, the recovery of bergenin in Sechangzhixiesan sample was in the range of $86.3 \%-92.3 \%(n=4)$. 


\section{Acknowledgments}

This study were supported by the Natural Science Foundation of Shandong Province (No. ZR2010BL025), Open Project of State Key Laboratory of Supramolecular Structure and Materials (No. sklssm201323)(Jilin University), State Key Laboratory of Inorganic Synthesis and Preparative Chemistry (No. 2011-13)(Jilin University).

\section{References}

[1] Yinxin He, Qiuling Zhang, Xiaochen Shi, Haiyan Wang, Xiaoliang Chen, Xiang Long, Journal of Medicine and pharmacy Minorities, 2014, 3, 62-64

[2] Yang Jin, Yan Wang, C hina Pharmacy, 2011, 22(7), 614-615

[3] Zhongqiong Li, Wenjie Zhang, Xin Ma, Xiong Yang, Chinese Journal of Pharmaceuticals 2004, 35(3), 170

[4] Ruyi Chen, Pei Luo, Yijie Li, Ting Zheng, Jiangke Qin, Journal of Guangxi Normal University( Natural Science Edition), 2015, 33(1), 99-102

[5] Zhibo Zhu, Qinglin Ma, Aizhen Liang, Jie Lu, Ligang Chen, Chinese Journal of Analysis Laboratory, 2013, 32(11), 108-111

[6] Ou Zhao, Yin Du, Hubei Agricultural sciences, 2016, 55(1), 186-188

[7] Jin Wang, Yan Ma, Zhanhong Wang, Yan Liu, Jing Wang, Yuejin Zhang, Journal of Northwest Forestry University, 2012,27(4), 186-189

[8] Ke Wang, Xiaqin Wu, Lin Li, Haitao Tian, Zhongqing Lu, Chemical Journal of Chinese University, 2013, 34(7), 1739-1742

[9] Xing Wang, Hua Zhao, Yingli Ma, Qiuchen Yang, Xiaoling Chen, Science and Technology of Food Industry, 2016, 37(2), 307-311

[10]Zewei Mao, Chunping Wan, Yuan Jiang, Gaoxiong Rao, Chemical Reagents, 2014, 36( 8), 689-692

[11]Wei Liu, Qiongmei Zhang, Journal of Hainan Normal University (Natural Science), 2011, 24(2), 177-179

[12]Xinyu Zheng, Lihui Zheng, Yongping Xie, Xiangyang Cai, Yulin Wang, Rixin Lv, Journal of Yunnan University, 2011, 33(2), 206-209

[13] Yan Wang, Yang Jin, Zhenqi Liu, Journal of Dali University, 2012, 11(9), 8-10

[14]Wenjie Hu, Xiaoying Dai, Bing Gong, Xiangmei Jiang, Jiangxi Forestry Science and Technologry, 2007, 5, 11-13 